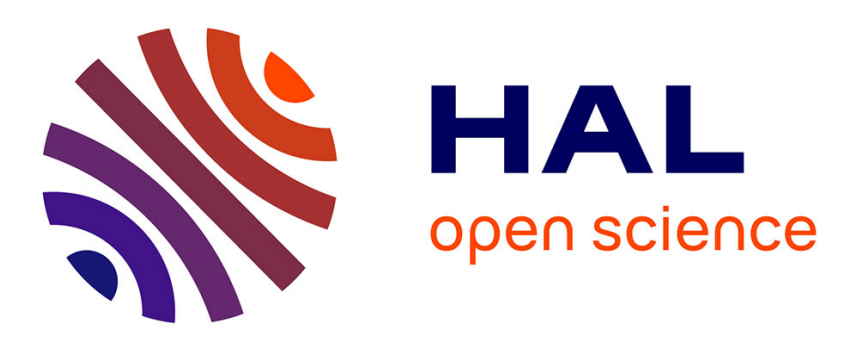

\title{
Accéder à un espace à soi. Contraintes de genre, luttes de classement et résistances en situation administrative précaire
}

Joanne Le Bars

\section{- To cite this version:}

Joanne Le Bars. Accéder à un espace à soi. Contraintes de genre, luttes de classement et résistances en situation administrative précaire. Annales de géographie, 2018. hal-02558314

\section{HAL Id: hal-02558314 \\ https://hal.science/hal-02558314}

Submitted on 29 Apr 2020

HAL is a multi-disciplinary open access archive for the deposit and dissemination of scientific research documents, whether they are published or not. The documents may come from teaching and research institutions in France or abroad, or from public or private research centers.
L'archive ouverte pluridisciplinaire HAL, est destinée au dépôt et à la diffusion de documents scientifiques de niveau recherche, publiés ou non, émanant des établissements d'enseignement et de recherche français ou étrangers, des laboratoires publics ou privés. 


\title{
Accéder à un espace à soi. Contraintes de genre, luttes de classement et résistances en situation administrative précaire
}

\author{
Access to a home. Constraining factors of gender, class \\ tensions and resistance in an insecure administrative \\ situation
}

Joanne Le Bars

Docteure en géographie, Lab'urba - Institut Émilie-du-Châtelet - ATER en géographie, Université Paris-Est Marne-la-Vallée

Résumé Cet article se propose d'interroger à partir de quatre «cas » la trajectoire résidentielle et la construction d'un chez-soi dans l'agglomération parisienne de femmes sans-papiers parties seules en France. Il souhaite montrer les variables sociales discriminantes de ces trajectoires résidentielles, de la construction d'un chez-soi et comment les rapports de genre, classe et " race » se forgent au quotidien, dans et par l'habitation, et participent à la définition de la position sociale de ces femmes.

Abstract This paper deals with housing trajectories and fighting to find a home among undocumented women who have migrated alone to the Paris region. It sheds light on the social differentiation between these women, their housing trajectories and their desires for a home. Factors regarding gender, class and "race" are shown to intermingle daily in and through the home to help define the social position of these women.

Mots-clés chez-soi, femmes sans-papiers, trajectoire résidentielle, déclassement social, classes populaires.

Keywords home, undocumented women, housing trajectories, social downgrading, working class.

Peu de recherches se sont penchées sur la trajectoire résidentielle, l'habitation et la question du chez-soi de femmes sans-papiers venues seules en France. Au cours de cette trajectoire migratoire, nombre d'entre elles se retrouvent évincées du marché locatif classique ou officiel et sont aux prises avec des lois migratoires qui conditionnent bien souvent l'accès au titre de séjour à des critères familiaux ou conjugaux (Lesselier, 2004). Alors que dans mon enquête ethnographique sur des femmes sans-papiers d'Afrique subsaharienne et du Maghreb en région parisienne (encadré l) je m'attendais à recueillir beaucoup de discours sur le travail ou l'administration, j'ai découvert combien l'enjeu de la résidence minait tout autant ces femmes.

Partant de ce constat, cet article se propose d'aborder la question de la résidence de femmes sans-papiers sous deux angles. D'une part, il s'agit, en adoptant une approche longitudinale, de poser la question des rapports de genre et plus largement de l'articulation des rapports de pouvoir (de genre, 
« race ", classe, âge) par et dans l'habitation. Comment les assignations de genre structurent l'habitation et plus largement la trajectoire résidentielle ? Comment le logement participe à reproduire, (re)configurer, faire vaciller les rapports de genre et les autres rapports de pouvoir ? Dans les logements des interlocutrices, au-delà des rapports de classe et de genre « qui s'entremêlent au quotidien » (Lambert, 2016), les rapports de "race» participent aussi à définir les positions sociales de ces femmes. Si dès les années 1970, les chercheuses féministes matérialistes ont souligné que l'espace privé ne pouvait être pensé en dehors des rapports sociaux de sexe et suscité des travaux adoptant cette perspective dans l'analyse de l'espace domestique, ces travaux sont beaucoup plus rares aujourd'hui (Lambert, Dietrich-Ragon, et al., 2014) ${ }^{1}$. Les études de genre privilégient l'étude de la sphère publique et politique (ibid.). C'est dans les recherches qui ont porté sur des situations de domesticité que la question des rapports de pouvoir entre hommes et femmes et entre femmes dans le logement reste posée ${ }^{2}$. Pour l'analyse des classes sociales, l'exploration du «foyer », du «logement », de «l'intérieur» ou de "l'espace domestique » est apparue comme une voie heuristique dans les années 1980 et 1990 en France, alors que depuis le début des années 2000, on

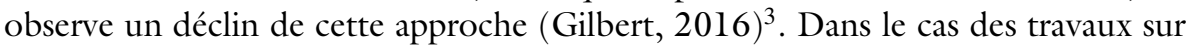
les situations migratoires en France, la question de la résidence n'est pas l'objet principal de recherche à l'exception de quelques travaux dans le cas de migration masculine et/ou familiale 4 . Au-delà du registre de la domesticité, concernant les femmes migrantes, des travaux ont abordé la question de la résidence mais en filigrane ${ }^{5}$. Deux recherches sont venues ouvrir de nouvelles pistes de réflexions

1 L'article d'Anne Lambert (2016) fait partie des rares articles qui croisent genre et classe pour penser I'habitation. En revanche, dans le contexte anglophone, notamment chez les géographes féministes, l'espace privé, la maison ont continué à être des objets d'analyse premiers (Moss, Dyck, 2003 ; McDowell, 2004 [1999] ; Pratt, 2004 ; Silvey, 2004).

2 Ils ont évoqué l'implication d'être logée chez son employeur pour les migrantes internationales (Pratt, 2004 ; Silvey, 2004 ; Oso Casas, 2005 ; Mozère, 2002 ; Drouilleau, 2002), pour les domestiques françaises et « bonnes à tout faire » de la bourgeoisie et petite bourgeoisie urbaine du XVIII ${ }^{\mathrm{e}}$ siècle au milieu du Xx $x^{\mathrm{e}}$ siècle (Perrot, 2009 ; Martin-Fugier, 1985 ; Fraisse, 1979) et les " petites bonnes » (jeunes filles) dans un contexte africain (Moujoud, 2011 ; Feldman, 2013 ; Jacquemin, 2000).

3 Les recherches sur les classes populaires se sont intéressées à la trajectoire résidentielle, aux intérieurs sans toujours les prendre comme objet premier d'analyse (Bourdieu, 1979; Schwartz, 2012 [1990] ; Weber F., 2013 ; Gilbert, 2016) et aux politiques publiques qui encadraient la résidence de ces groupes sociaux (Girard et al. 2013 ; François, 2014).

4 Parmi ces recherches, $c^{\prime}$ est davantage la question de la résidence à un moment donné de la trajectoire qui est posée (Razy, 2006 ; Timera, 1997 ; Mbodj-Pouye, 2016 ; Delage, Weber, 2014) ou la gestion administrative et politique des immigrés postcoloniaux qui est analysée à partir des foyers de travailleurs (Bernardot, 2008 ; Hmed, 2006 ; Béguin, 2015 ; Samuel, 1978 ; Sayad, 1980), des centres de rétention (Fischer, 2010 ; Makaremi, 2010 ; Bernardot, 2008) ou l'angle de la ségrégation résidentielle qui est privilégié (Poiret, 1996 ; Verdugo, 2011) et non la trajectoire résidentielle qui est posée (À l'exception de Simon, 1995 ; Têtu-Delage, 2009) et encore moins la question du chez-soi et des rapports de genre dans le logement.

5 Sur les femmes d'Afrique subsaharienne vivant en France dans un cadre polygame (Fainzang, Journet, 1988 ; Nicollet, 1992) et sur les migrantes marocaines parties seules à Paris (Moujoud, 2007). 
sur les trajectoires résidentielles de migrantes ${ }^{6}$. Par ailleurs, le travail de Camille Schmoll (2005), adoptant une approche interscalaire et genrée, a montré les ressources que constituaient les espaces privés et notamment les hôtels pour les commerçantes tunisiennes en Italie.

D'autre part, dans cet article, il s'agit de poser la question du chez-soi. Toutes les interlocutrices de mon enquête n'ont pas connu une trajectoire résidentielle en France ponctuée par les dispositifs d'hébergement d'urgence («115 », encadré $2)^{7}$. Ici, je m'intéresserai donc à des femmes qui n'ont jamais connu le « 115 », d'autres qui ont connu le «115» et qui ont fini par réussir à trouver une alternative à celui-ci. Cette expérience de passer de l'absence de chez-soi à la possession d'un chez-soi est alors particulièrement opportune pour saisir ce qui apparaît pour les interlocutrices comme le «luxe» d'un chez-soi et poser la question des possibilités de ces trajectoires qui échappent au « 115 ». La question du chez-soi est posée par des travaux de géographes français sur «l'espace domestique »(Collignon, Staszak, 2004), chez les pionniers anglophones des approches à une micro-échelle (Tuan, 2014 [1977]) et les géographes féministes (Pratt, 2004 ; Moss, Dyck, 2003). Pour croiser la question du chez-soi, il faut également chercher dans la littérature sur des hommes et des femmes dans des situations d'extrême pauvreté avec des sans-abri en France ${ }^{8}$, dans des contextes "d'habitat en situation limite ${ }^{9}$ ". Le croisement de ces cas dans le cadre d'une enquête et la considération de l'espace vécu et perçu par les interlocutrices nous permettent de mesurer ce qu'est un chez-soi : un lieu relativement stable, un lieu à soi marqué par l'habitude et l'ancrage (Lion, 2014), un espace physique approprié qui préserve de l'extérieur et sur lequel la résidente a une autorité ${ }^{10}$ mais qui est aussi marqué par l'expérience (Tuan, 2014 [1977]), par des pratiques associées (les commerces, les habitudes de consommation qui y sont liées, une décoration, un agencement...), un ensemble de personnes qui y passent, de souvenirs qui se construisent. L'existence d'un chez-soi revient ainsi à se préserver un espace autonome personnel pour ne pas «perdre la face » (Goffman, 1973), et perdre son «identité sociale» (Bourdieu, 1978).

Les quatre «cas » sur lesquels je m'appuie ici - ceux de Fatoumata, Naïma, Lalia et Nadra ${ }^{11}$ - sont autant $d^{\prime}$ " expériences sociales ». Chaque cas singulier est

6 Laura Oso Casas a souligné les multiples configurations des trajectoires résidentielles des migrantes espagnoles (2005) à Paris et Florence Bouillon a retracé la trajectoire résidentielle d'une femme malienne (2011).

7 Sur 52 interlocutrices, 21 ont eu à être prise en charge par le « $115 »$.

8 Girola, 2011 ; Zeneidi-Henry, 2002.

9 Comme les habitants du bois de Vincennes (Lion, 2014), dans le cadre de logements de passage en France (Lévy-Vroelant, 2000) que ce soit des squats (Bouillon, 2005) ou des hébergements sociaux (Thalineau, 2002), dans l'espace cellulaire en prison (Bony, 2015).

10 Ces réflexions renvoient à celles posées sur "l'intimité » par Jean-François Laé et Bruno Proth (2002).

11 Les noms et lieux ont été anonymisés en raison du contrat moral qui me lie aux enquêtées. Dans cet article, l'utilisation du seul prénom vise à rendre compte de la relation ethnographique tissée, soit une relation proche. En ce qui concerne les lieux évoqués (commune, quartier, etc.), j'ai cherché à garder le même profil social des lieux en trouvant des équivalents sociaux. 
compris comme le croisement de multiples histoires collectives et « la comparaison des cas permet d'établir ensuite les domaines de validité de ces histoires collectives et la probabilité de leur intersection » (Weber, 2013, p. 36-37) offrant des mécanismes sociaux qui se reproduisent ailleurs. Dans les sillons d'Alban Bensa (2006), j'ai choisi d'accorder à la description une place centrale dans l'analyse.

Le premier temps de cet article s'intéresse à Naïma lorsqu'elle est confrontée au « 115 », un portrait qui permet d'étudier la façon dont, dans des contextes d'hébergements collectifs contraints, cette enquêtée tente de préserver un espace à soi et les rapports de pouvoir qui se jouent dès lors entre femmes. L'emménagement d'une autre interlocutrice, Fatoumata, dans un «studio » en cohabitation conjugale au cœur d'un squat d'un quartier populaire de l'agglomération parisienne, constitue le deuxième temps de cet article. Cette nouvelle résidence permet de souligner les conditions d'extraction du «115», de s'interroger sur ce que peut être un chez-soi, de s'intéresser aux ressources que peut constituer le fait d'habiter dans un quartier populaire et les rapports sociaux de sexe qui se jouent dans ce logement. Enfin, dans un troisième temps, je me concentre sur Lalia et Nadra, l'une locataire d'une chambre de bonne dans le $\mathrm{XVI}^{\mathrm{e}}$ arrondissement de Paris et l'autre d'un «studio » d'un quartier du centre de la ville, toutes deux vivant en dehors de la cohabitation conjugale. N'ayant jamais été confrontées aux structures d'hébergement du " 115 », ces deux enquêtées dont la trajectoire sociale diverge (l'une est issue des classes populaires et l'autre de la petite bourgeoisie culturelle) permettent d'identifier les ressources sociales et économiques nécessaires au maintien du chez-soi dans la trajectoire migratoire. Ces deux derniers « cas » soulignent également les rapports de force qui se jouent à l'échelle de l'immeuble avec des femmes d'autres groupes sociaux.

\section{L'ENQUÊTE ETHNOGRAPHIQUE}

L'enquête qui s'est déroulée dans le cadre d'un doctorat (Le Bars, 2017) jusqu'en 2017 a commencé fin 2009 dans deux lieux militants situés dans un quartier populaire de l'est parisien : une occupation (Les Amandières) - squat d'activité et d'habitation - initiée par $d^{\prime}$ anciens sans-papiers et une association engagée dans la cause des sans-papiers (Droits des Sans-Papiers). Suite à l'occupation des Amandières, $\mathrm{j}^{\prime} \mathrm{ai}^{12}$ été dirigée, par quelques interlocutrices de ce lieu, vers des institutions privées ou publiques de l'urgence sociale ou de l'aide sociale : un lieu d'accueil de jour pour les femmes sans-abri, un centre d'hébergement d'urgence, un centre d'hébergement et de réinsertion sociale, une association qui se présente comme étant « communautaire » œuvrant dans le domaine de la santé envers les immigrés. Ces structures encadraient au quotidien une partie des femmes rencontrées aux Amandières après l'évacuation du lieu. Si 52 interlocutrices (28 femmes d'Afrique subsaharienne et 24 femmes du Maghreb) ont été rencontrées à plusieurs reprises sur différentes scènes sociales (militante, résidentielle et du travail), 14 interlocutrices constituent le noyau dur de l'enquête. Parties seules de leur pays d'origine avec, pour la majorité d'entre elles un visa touristique, ces femmes sont célibataires,

12 Jeune femme blanche hétérosexuelle issue de la petite bourgeoisie « contre-culturelle » d'une ville moyenne. 
divorcées, veuves. Elles sont issues de différents groupes sociaux et âgées de 24 à 70 ans.

\section{LE « $115 »$}

Le « 115 » est un numéro de téléphone administré à Paris par le Samu social. Le Samu social de Paris, Groupement d'intérêt général dont l'État est le principal financeur, fondé en 1994, est dans le domaine strict de l'urgence (Cefaï, Gardella, 2011). Cette structure administre un grand nombre de dispositifs et tisse des partenariats avec de nombreuses associations notamment en ce qui concerne les structures d'hébergements et d'accueil de jour. L'usage du terme « 115 » pour les femmes que j'ai rencontrées recouvre l'ensemble des structures qui les héberge la nuit pour une durée d'un jour à plusieurs semaines et l'ensemble de celles qui les accueillent la journée. Dans leurs discours, elles ne font pas apparaître les différences de statuts entre ces institutions (privées ou publiques). Les distinctions qu'elles opèrent entre les structures reposent sur leurs expériences pratiques de celles-ci. En France l'hébergement d'urgence est possible pour les personnes quel que soit leur statut administratif, à l'inverse d'autres pays d'Europe (Sprakel, 2010, cité par Oppenchaïm, Le Mener, 2012). L'augmentation des demandeurs d'asile et des sans-papiers parmi les sans-abri en France et dans les États de l'Europe, modifie le profil de ces derniers (Bursch-Geertsema et al., 2010 cité par Le Mener, Oppenchaïm, 2012), dans un contexte d'engorgement du système d'hébergement d'urgence. En France métropolitaine, pour les agglomérations de 20000 habitants ou plus, les femmes représentent $38 \%$ des personnes sans-domicile et parmi celles nées à l'étranger, elles sont souvent hébergées en hôtels ${ }^{13}$ (Yaouancq et al., 2013).

\section{Quel chez-soi face au « 115 »? Pratique pieuse et bonne volonté culturelle}

Je fais la connaissance de Naïma à l'occupation des Amandières. De ce fait, nous nous sommes vues un très grand nombre de fois. Elle me demande régulièrement des nouvelles de l'avancée de ma thèse. Âgée de 50 ans au moment où je la rencontre et enseignante lorsqu'elle résidait en Algérie, elle met souvent au-devant de la scène la position sociale qu'elle possédait dans son pays d'origine. Elle me rappelle souvent le niveau d'étude de ses deux sœurs et le métier qu'elles exercent (enseignantes), ainsi que le statut de son frère ingénieur marié à une médecin qui réside en France. Elle trouve peu d'heures de travail et essentiellement dans la garde d'enfants et le ménage chez des particuliers.

\subsection{De l'entraide familiale au dégagement de la domination du frère}

À son arrivée en France en 2007, elle est accueillie par son frère et sa compagne dans la région parisienne pendant un an et demi. La cohabitation est très difficile

13 Pour les personnes francophones de 18 ans ou plus (enquête Ined, Insee, 2012 ; Yaouancq et al., 2013). 
m'explique-t-elle, elle est marquée par des réflexions et un contrôle de ses pratiques quotidiennes au sein de la maisonnée et en dehors :

«[C]'était infernal avec lui, infernal, infernal, il était méchant. Ab oui, il te faisait des réflexions? Des réflexions: "Faut pas rentrer après 8 heures, 8 heures 30, y'a pas de travail le soir". Donc, il me cherchait la p'tite bête, pour par exemple la lumière : "Il faut pas allumer trop de lumière". Euh la douche il faut le strict minimum de l'eau pour la douche. » (Entretien, le 15 mars 2010 dans sa chambre aux Amandières)

Habiter chez son frère qui impose son pouvoir ne peut constituer pour Naïma un chez elle. Elle vit comme un rejet et une agression le comportement de son frère. Ajoutée à un travail en rien lié à ses études universitaires et au processus de déclassement qui en résulte, la situation au sein de la maisonnée de son frère lui paraît invivable. Et finalement Naïma part. Elle est alors prise en charge par les dispositifs d'hébergement d'urgence. Cette instabilité familiale engendre un appauvrissement et une instabilité résidentielle dans un contexte déjà difficile.

\subsection{Le sac et les menus objets du quotidien pour s'approprier les chambres $d u \ll 115 »$}

Naïma a toujours valorisé le capital culturel qu'elle possède notamment en faisant référence à ses études et à son goût pour la lecture. La description qu'elle me fait de ce sac qui l'accompagne toujours dans ses déplacements dans les structures d'hébergement d'urgence est révélatrice à ce sujet. Venue me rendre visite chez moi, elle tient à me montrer ces menus objets qui meublent son quotidien dans ces structures. Elle transporte toujours au moins deux livres : son Coran (le petit me spécifie-t-elle) et un roman. Elle « lit quelques versets coraniques » pour « $[\mathrm{s}] \mathrm{e}$ remonter le moral » (photographie 1).

Elle emporte également toujours un petit tapis de prière dans son sac : "Mon tapis est là, tout le temps avec moi, mon petit tapis. Il est petit, il est mignon, c'est ma mère qui l'a ramenée de La Mecque. Un cadeau ». Pour elle c'est « très important » de «faire [s]a prière ». Elle m'explique : «Bon moi $j$ 'dis pas que j'suis pratiquante à $100 \%$ mais bon le minimum qui exprime ma foi, c'est tout ». Elle me fait part de la difficulté à tenir la conduite pieuse qu'elle souhaiterait dans les centres d'hébergement d'urgence. Elle écoute à l'aide de son téléphone portable différentes radios dont Radio Orient et tient à me faire écouter lors d'un entretien (le 15 août 2012 chez moi) les invocations après l'appel à la prière qui rythment son quotidien. Elle relate combien la réitération de pratiques corporelles pieuses l'apaise dans ce quotidien (les ablutions, le moment de la prière, etc.). Dans son sac, elle a toujours également "un petit savon », du shampoing, une brosse à dents, le médicament qu'elle prend depuis un ulcère à estomac et «un rouge à lèvres, un petit crayon et un petit fard à joues comme toutes les filles ». "Avec le "115" on grossit» me dit-elle ou encore « Il faut faire attention à son corps ». Beaucoup des interlocutrices parlent de cette influence directe de l'espace d'hébergement contraint sur le corps, par la nourriture contrainte, par la 


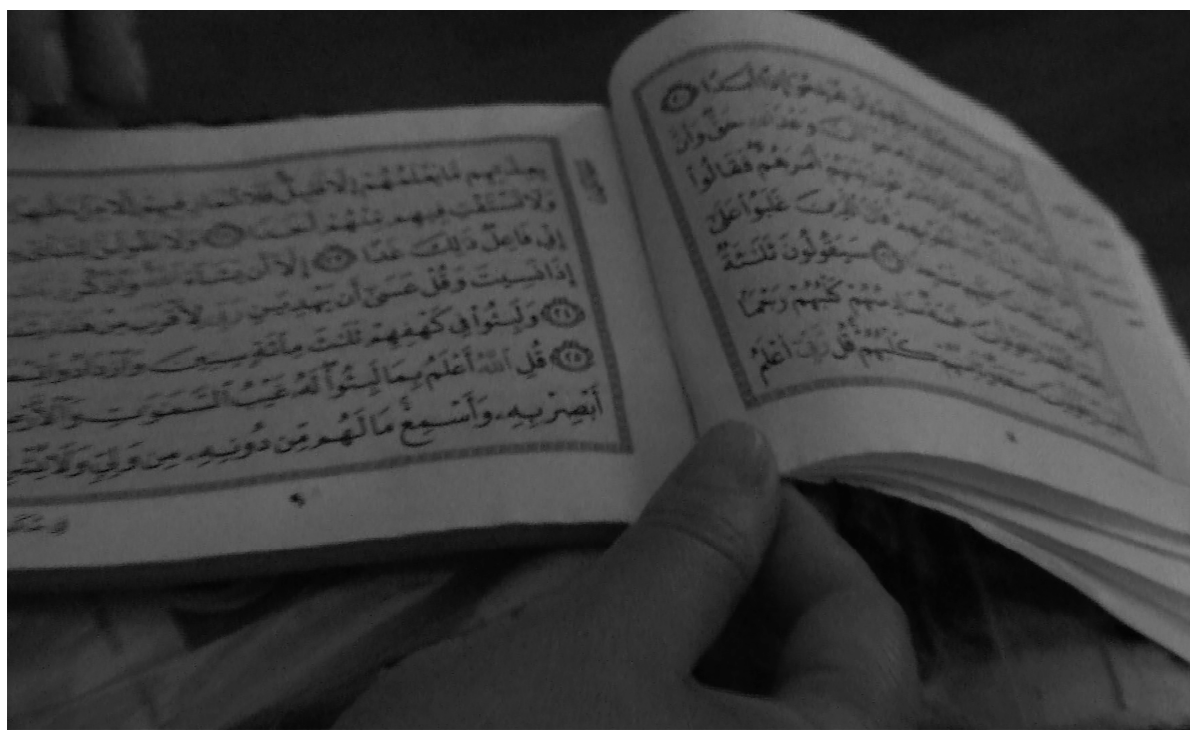

Source : cliché de l'auteure, 2011.

Photo 1 Le «petit »Coran de Naïma. Naïma's "little" Coran.

fatigue des déplacements, par les petits renoncements à son hygiène de vie. Elle emporte également des draps parce que «leurs draps, ils sont pas agréables » me dit-elle, «ils glissent » et «ils sont pas en coton». Le reste de ses bagages est chez son frère et « chez une personne chez qui elle a travaillé avant ». L'ulcère à l'estomac est dû au stress d'après le médecin, me dit-elle. Elle met ce stress sur le compte de son quotidien actuel marqué par l'incertitude de l'hébergement :

«[...] chaque fois avec le " 115 " on n'a pas le droit de rester plus d'une semaine et des fois on te donne deux jours, trois jours et des fois quand tu rappelles il faut rappeler, $[\ldots]$, y'a un monde de fou $[\ldots]$. On est perturbé, psychiquement on n'est pas bien, on peut même pas chercher du boulot ou faire des courses comme tout le monde pourquoi parce qu'on est là avec sa valise, $[\ldots]$ et puis là des fois le " 115 ” jusqu'à 19 heures ils te répondent pas, ils te répondent le soir et ils te disent : “On a plus de place”. C'est saturé, c'est fermé, donc il faut prendre ta valise et aller à l'hôpital, rester sur une chaise, les urgences... »(Entretien, le 15 août 2012 chez l'enquêtrice).

La description des pratiques de Naïma dans le contexte du « 115 » nous montre que celles-ci sont des formes de protection et de lutte quotidienne qui s'incarnent dans les corps (prendre soin de son corps, se laver, maîtriser son corps, etc.) et dans les pratiques d'appropriation de l'espace des chambres qu'on leur attribue (disposer ses menus objets du quotidien, laver avec de la javel la chambre, etc.). Pour certaines femmes, le maintien d'une conduite pieuse, « d'une 
subjectivité vertueuse » (Mahmood, 2009) dont les formes de religiosité sont pour chacune différentes, apparait comme une structure quotidienne indispensable, protectrice, vectrice d'apaisement. Cette conduite pieuse agit à la fois comme maintien de soi, comme une mise en quotidien et une structure du temps ritualisé. De même, le sac qui les accompagne dans ces hébergements d'urgence peut constituer une ressource. Si dans le contexte du «115 », j'ai analysé certaines de ces pratiques comme des formes de protection, elles peuvent aussi constituer des formes de distinction sociale.

\subsubsection{Se distinguer des autres hébergé.e.s pour symboliquement " ne pas être là "}

Naïma cherche à se distinguer des autres hébergé.e.s dans les conversations qu'elle a avec moi. Cette distinction prend corps sur la question du travail, celui auquel elle est assignée : la pénibilité du cadre et du rythme de travail, l'insécurité de l'emploi, les heures mal payées. «Moi j'accepte tout» me dit-elle ou encore «je travaille dans n'importe quoi ». Elle décrit la dispersion des heures et des lieux de travail. Elle souligne sa "bonne volonté » par rapport à certains " Français de souche » qui sont également hébergé.e.s par les structures du «115»: «Y’a des Français qui sont dehors parce qu'ils choisissent, ils ne veulent pas travailler dans n'importe quoi ». Naïma effectue également des formes de distinctions par rapport aux autres hébergées qui reposent sur les titres scolaires. L'attention qu'elle porte à rappeler son ancien métier et les diplômes qu'elle possède et la forte reconnaissance sociale qu'elle donne au diplôme universitaire (le doctorat) que je prépare, marque une relation d'admiration à l'école et aux diplômes scolaires. Elle croit à la culture légitime et au salut par l'école. Ce processus de distinction se met aussi en place à travers le registre de la propreté. Elle me raconte par exemple à propos de la Halte Simone de Beauvoir qu'elle «nettoyai[t] les WC avant d'y aller, les femmes avaient peut-être le sida », et qu'elle y lavait «aussi les tables ».

Naïma vit cette expérience avec le " 115 » comme une injustice, une nonreconnaissance de sa «juste valeur». À travers ces propos se lit sa lutte contre le déclassement. Le déclassement qu'elle subit avec la migration, déclassement marqué notamment par sa trajectoire résidentielle, lui est extrêmement difficile à vivre comme pour beaucoup de femmes que j'ai rencontrées. En condamnant moralement les autres résidents, en dénonçant leurs pratiques afin de se positionner comme socialement supérieure, Naïma cherche surtout à me et à se convaincre qu'elle appartient plus à mon monde universitaire qu'à celui de l'hébergement d'urgence.

\subsubsection{La mosquée, lieu de sociabilité, d'ancrage et de ressources genrées}

Pendant toute la période où Naïma est hébergée dans des centres d'hébergement d'urgence, la Mosquée de Paris est un lieu qu'elle fréquente assidûment. Ce lieu cultuel lui permet de faire ses prières ("J'vais là-bas pour faire ma prière parce que j'ai pas où la faire ») notamment de suivre la prière collective et de faire des rencontres féminines qui l'apaisent par le soutien qu'elles constituent. Un lieu 
qui permet «de s'entraider» entre femmes comme elle l'énonce. Des liens se tissent notamment du fait de la proximité cultuelle et sociale des femmes qui fréquentent ce lieu : "J'ai extériorisé, j’ai trouvé des sœurs, qui m’ont écoutée, qui m'ont relaxée et voilà » ou encore "J'ai raconté ma situation, elles m'ont soulagée, j'étais en pleurs, en stress et tout ça. » Une femme, rencontrée à la mosquée, lui propose de "donner des cours d'arabe à des enfants " dans une association tous les dimanches. Elle est heureuse : si ce travail est faiblement rémunéré, il lui apporte une très forte reconnaissance sociale au regard de sa trajectoire.

La fréquentation par les interlocutrices d'autres lieux protecteurs (comme les mosquées par exemple pour Naïma), le renforcement du réseau amical notamment féminin, leur très fine connaissance du tissu associatif à l'échelle de Paris, sont autant d'atouts pour se préserver soi quand on n'a plus de chez-soi. Ces formes d'appropriations sont élaborées à des fins de constructions personnelles.

Ce passage par le « 115 » et la disqualification sociale qui en découle matérialise, pour les interlocutrices issues des franges des classes populaires stabilisées ou des classes moyennes dans leurs pays d'origine, un processus de déclassement social. Lors de nos rencontres, elles mettent en avant la violence institutionnelle qui régule leurs pratiques jusqu'au plus intime, celle de la chair. Elles puisent dans leurs normes sociales et leur socialisation les moyens de se distinguer, de s'échapper de l'espace physique où elles dorment qui ne peut être un chez-soi. Quand on n'a pas d'espace à soi et que les lieux de restauration de son corps, du sommeil, de la toilette, sont des lieux devant être partagés par les autres, l'agression sur la personne peut être très forte. Ces femmes cherchent ailleurs dans la ville, dans les lieux de culte, dans les réseaux de sociabilités féminins, des espaces de repos de soi qui puissent un peu les préserver de cette violence qu'elles subissent.

\section{Enfin un chez-soi... en cohabitation conjugale. Les conditions d'extraction du « 115 »}

L'installation de Fatoumata dans un « studio » d'un quartier populaire offre un contrepoint exemplaire à l'expérience de la mobilité contrainte extrême engendrée par le « 115 » et à l'importance d'avoir un espace à soi, un chez-soi stable. En cohabitation conjugale, ce logement est traversé par des rapports sociaux de sexe qui tendent entre autres à reconfigurer ses pratiques, celles d'une femme qui auparavant vivait seule, en colocation ou dans des espaces collectifs. 


\section{1 "Mon château " un studio sans fenêtre dans un quartier populaire}

Quelques mois après l'évacuation du squat des Amandières, fin 2010, Fatoumata emménage avec Ousmane dans "un studio » ${ }^{14}$ situé dans un quartier populaire d'une commune à la limite de Paris. Encore «sans-papiers » au début de cette nouvelle résidence, elle sera régularisée quelques mois plus tard. Depuis 2007, Fatoumata a un emploi dans la garde d'enfants avec une même famille qui la rémunère au Smic. Elle reçoit un salaire de 770 euros en moyenne par mois. Quant à Ousmane, il a été régularisé pendant l'occupation des Amandières. Depuis que je le connais, il travaille dans le bâtiment. Fatoumata, originaire des franges les plus stables des classes populaires citadines du Mali, est âgée de 53 ans au moment où je la rencontre aux Amandières. Elle était aide-soignante au Mali, mère d'une fille dont elle a divorcé du père quelques années avant son départ pour la France. Arrivée en 2003 en France, elle a également été prise en charge par le «115 » pendant quelques mois dans sa trajectoire résidentielle.

\subsection{Le "château ", I'immeuble et le quartier}

Un grand porche, avec une porte noire qu'il suffit de pousser pour entrer, sépare la rue de l'ensemble d'immeubles où se situe le studio de Fatoumata. Une cour relie les deux immeubles qui composent cet îlot. À chacune de mes visites, les espaces communs étaient occupés par des groupes d'âge et de sexe différents tous originaires d'Afrique subsaharienne. Les immeubles sont particulièrement vétustes. Ousmane et Fatoumata m'ont raconté avoir eu accès à ce logement par le biais de «jeunes arabes du quartier» contre la "modique » somme de 2000 euros. Ils n'ont pas de bail et m'ont ensuite expliqué ne rien devoir payer en termes de loyer. C'est par l'intermédiaire d'Ousmane que l'obtention de ce logement s'effectue.

Fatoumata parle de cette habitation en ces termes:

«Ici, ouh là là, je me sens très, très, très bien ici, c'est mon château. Je vais, je viens quand je veux sans sonner à la porte, donc c'est le plus grand bonheur en France. Avec un grand lit de trois places, de l'eau chaude, ma cuisine, tout, les toilettes, tout, j'ai tout à ma disposition. » (Entretien, le 26 août 2012 , dans son «studio »)

"C'est mon château » : le possessif ici montre déjà que ce lieu peut être le sien, à la différence des chambres du " $115 »$. C'est bien chez elle puisque pour une fois, elle n'a pas à sonner pour y rentrer. C'est elle qui désormais contrôle le flux de l'entrée. La notion de "château ", ici bien sûr ironique, montre combien pour cette femme sa condition de résidence, c'est-à-dire avoir un chez-soi certes bien modeste, mais qui ramène en fait à la situation d'avoir un domicile à soi et pour

14 Ousmane a deux autres épouses. Une des épouses vit au Sénégal, l'autre dispose d'un logement avec ses enfants à Paris. Ousmane vit une semaine chez Fatoumata et l'autre semaine chez l'épouse qui vit à Paris. 
soi, est nettement plus favorable que les solutions d'urgence. Son " château » est un luxe inestimable de protection de soi, de construction de sa personne et de son estime de soi. Situé au rez-de-chaussée du deuxième immeuble, son «studio » d'environ $15 \mathrm{~m}^{2}$ ne possède pas de fenêtre. Il a une salle de bains où se trouvent également les toilettes et un petit espace pour la cuisine avec des plaques électriques, un réfrigérateur et un évier. L'enthousiasme avec lequel Fatoumata parle du fait d'avoir sa salle de bains et ses toilettes souligne combien la privation de ces espaces avec le « 115 » a pu être une souffrance. De même, elle accentue le possessif pour dire «Ma cuisine », marquant enfin la possibilité de reprendre la main sur son alimentation. Un rideau en tissu épais est accroché au-dessus de la porte d'entrée pour protéger du froid. Cette porte n'est jamais fermée la journée, elle reste entrouverte. Régulièrement, d'autres habitant.e.s de l'immeuble entrent chez elle pour la saluer. Quand je viens lui rendre visite, la télévision est allumée. Ousmane et elle sont friands des séries françaises, états-uniennes et nigérianes (avec la télévision, le chez-soi est aussi un espace de divertissement et de culture).

J'ai connu plusieurs transformations de la chambre de Fatoumata. Il y a d'abord eu le lit : il a été remplacé par un plus grand, un lit «trois places » me dit Fatoumata. Puis il a été déplacé : il a pris la place de la télévision et de l'armoire. Ensuite, un rideau a été installé pour séparer en deux la chambre, donnant l'illusion de plusieurs pièces : une partie pour le lit et l'autre pour «le salon ». Cette pratique d'aménagement divise l'espace en plusieurs lieux. Fatoumata change régulièrement de rideaux, elle en possède de différentes couleurs. Des chaises (six montrant la volonté d'accueillir du monde) ont été achetées pour consacrer ce «salon» qu'elle appelle en ma présence «le salon des pauvres». Cette nouvelle configuration de la pièce permet que «les gens soient plus à l'aise » m'explique-t-elle. En effet : «Ils partaient vite parce qu'il fallait passer contre le lit pour aller au fond de la chambre pour enfin s'asseoir, ils se gênaient un peu!» Il est vrai que cet agencement procure l'impression d'être dans un espace plus vaste. Avoir un chez-soi, contrairement à une chambre du « 115 », c'est retrouver la possibilité de recevoir, d'avoir une sociabilité choisie, d'être maître des mouvements chez-soi. Le studio est petit, une seule pièce, mais l'agencement cherche à produire différents espaces, comme si on vivait dans un appartement. La chambre est bien un chez-soi qui remplit toutes les fonctions primaires d'une «vraie » maison, dormir, cuisiner, se laver, recevoir, se divertir, généralement marquée par une division nette de l'espace et des pièces. Un grand tapis de la taille de la moitié de la pièce habille le salon. Il marque la frontière avec l'entrée, cette limite à partir de laquelle il faut se déchausser. Fatoumata et Ousmane effectuent leur prière dans cette partie de la pièce. La petite télévision qui campait sur le meuble de la chambre est aujourd'hui remplacée par un écran beaucoup plus grand. Les affiches et photos accrochées sur les murs se sont multipliées. Ce sont pour la plupart d'entre elles des représentations de la famille d'Ousmane et d'Ousmane seul. Cette maison est celle d'un couple et pas seulement de Fatoumata, l'espace porte donc aussi la marque personnelle de son mari, lequel a souhaité y mettre en scène sa famille. Avoir un chez-soi c'est 
donc aussi pouvoir mettre en scène ses filiations choisies, se réinscrire dans son histoire par le choix des objets personnels dont les photos sont une expression. Un calendrier musulman donné par un boucher halal et une horloge complètent le décor mural. Tous ces objets marquant une histoire personnelle, décorent et habillent le chez-soi. Ils permettent visuellement de se dire qu'ici c'est bien chez-soi. Ici, on ne parle plus de javel, il n'y a plus à se protéger du lieu, le lieu est devenu à soi. L'ensemble des dépenses et des travaux d'installation du studio a été assuré par Ousmane. Il achète un grand nombre de meubles et d'objets sur le marché des biffins ${ }^{15}$ situé à 200 mètres de leur immeuble. L'agencement se fait donc dans une économie de la débrouille, la seule ouverte pour les pauvres.

Cette stabilité résidentielle qu'a pu obtenir Fatoumata (une stabilité dans le contexte français qui reste cependant très fragile) se lit dans son intérieur à la fois par les objets et meubles qui y sont disposés et par les transformations effectuées dans la chambre. Son intérieur dévoile sa position sociale au Mali et le déclassement social qu'elle vit en France. Les nouveaux aménagements engagés au fil du temps dans son logement reflètent les aspirations de Fatoumata : celles de retrouver la position sociale qu'elle avait au Mali. "C'est le salon des pauvres!» reflète de manière explicite à la fois cette expérience du déclassement vécue avec la migration et sa volonté d'ascension sociale (et par exemple d'avoir un salon pour recevoir ses amis).

Fatoumata a une forte mobilité dans son quartier et hors de celui-ci. Pour acheter les produits alimentaires et également d'autres menus objets elle se rend dans différents lieux du quartier (marché, bouchers halal, Lidl, Monoprix). Elle visite régulièrement des membres de sa famille les week-ends dans la banlieue parisienne. Elle participe également à des cérémonies ${ }^{16}$ et aux rassemblements militants de l'ancienne occupation des Amandières. Son travail engendre des trajets quotidiens dans un arrondissement du sud de Paris. Quand je pars de chez Fatoumata en sa compagnie, sur le trajet, nous croisons toujours, dans son immeuble et dans les trois rues qui mènent à l'arrêt de bus, des gens qu'elle connaît (tous originaires d'Afrique de l'Ouest).

\subsection{Un chez-soi comme espaces de sociabilités, d'ancrage et de ressources}

Au fil de mes visites dans son "château ", j'ai pris conscience de l'importance de cette scène locale pour elle. Le quartier se révèle ici comme une ressource de proximité. Les économies quotidiennes engendrent pour Fatoumata des mobilités dans des lieux très précis et délimités du quartier populaire de la commune où elle habite. Il y a également la densité des relations sociales nouées au niveau de la scène locale (les réseaux sociaux amicaux et de voisinage localisés), leur

15 Les biffins sont des personnes qui vendent des objets de récupération. Le marché des biffins fréquenté par Fatoumata et Ousmane est un marché qui a été institutionnalisé. Les vendeurs doivent adhérer à une charte pour pouvoir bénéficier d'un emplacement.

16 Ces évènements (naissance, mariage, etc.) sont le socle d'échanges cérémoniels féminins très importants pour les femmes d'Afrique de l'Ouest. 
quotidienneté pour certaines ou leur régularité pour d'autres qui engendrent une maîtrise et une connaissance de l'espace local (Fol, 2010). Fatoumata a également pu développer un commerce de sacs à main et de maïs grillés. La possibilité de stocker des marchandises dans son "studio » et la localisation du logement dans un quartier populaire ont participé à rendre possible cet entreprenariat commercial (Le Bars, 2017). J'ai compris que le statut local de Fatoumata à l'échelle de ce quartier et le capital social qu'elle en retirait, pouvaient participer à relativiser son statut professionnel (déclassé et situé à l'extérieur de ce quartier). Pour Fatoumata, dans un contexte de "multi-appartenances » fondées sur l'éclatement des scènes sociales, cette nouvelle résidence permet d'ouvrir l'espace social sur une autre scène que celle du travail (Chamboredon et al., 2009 [1985]). Ce statut local lui offre ainsi une protection et une force nouvelle.

\subsection{4 Un chez-soi comme tremplin mais traversé par des rapports sociaux de sexe}

L'intérieur de la chambre de Fatoumata révèle les rapports sociaux de sexe qui s'y nouent dans un modèle conjugal précis : un couple en cohabitation une semaine sur deux. "C'est la chambre d'Ousmane » répond Fatoumata à ma question sur les photos et affiches accrochées sur les murs, lesquelles portent essentiellement sur son époux et sa famille. Elle ne prononcera qu'une seule fois cette assertion et pourtant, si Fatoumata vit et parle constamment de "sa chambre ", "son studio ", "son château ", et qu'elle donne son avis sur l'aménagement de la pièce, l'appropriation des murs par Ousmane matérialise le rapport de sexe inégalitaire qui les unit : Fatoumata est dans l'unité domestique d'Ousmane. Cet intérieur souligne également le travail engendré par la recherche des économies quotidiennes, un travail qui repose sur des pratiques genrées. Fatoumata est chargée de la recherche des prix les plus bas en ce qui concerne les denrées alimentaires, Ousmane se charge de la recherche du meilleur prix pour ce qui relève notamment des meubles pour l'aménagement du logement. L'ensemble des frais est assuré par Ousmane ${ }^{17}$. Le temps de Fatoumata hors du travail rémunéré dans le service domestique s'organise, une semaine sur deux, dans les tâches ménagères pour le couple (ménage, courses, cuisine, etc.). Ousmane et Fatoumata tiennent un discours commun sur le caractère "naturel » et " complémentaire » de la division sexuée du travail comme le soulève Anne Lambert (2016, p. 64) à propos des fractions stables des classes populaires et petites classes moyennes enquêtées. Cependant, ce discours sur la complémentarité des rôles mis en avant par Fatoumata, "cache mal la répartition déséquilibrée des tâches au prestige et à la visibilité inégales » (ibid.).

Au Mali, Fatoumata était dotée d'une forte reconnaissance sociale notamment dans son quartier. Sa position sociale au Mali (sa qualification professionnelle, son

17 Jeanne Semin (2007) souligne qu'en contexte migratoire, les femmes originaires d'Afrique de l'Ouest en couple refusent de participer aux frais du foyer (loyer, factures, vêtements, courses alimentaires, etc.) même si elles ont pour beaucoup d'entre elles un revenu lié au travail qu'elles exercent. 
capital social, etc.), sa trajectoire sociale en France (son remariage, son inscription dans les réseaux militants, son capital social à Paris, un emploi « stable » selon elle et rémunéré au Smic), tendent à expliquer son ancrage réussi dans un quartier populaire tout comme ce rapport positif au quartier. Cette nouvelle résidence en cohabitation conjugale participe à reconfigurer les pratiques de Fatoumata. Si les tâches domestiques occupent une partie de son temps hors du travail salarié, son âge (53 ans) lui permet d'échapper à une emprise totale des tâches domestiques sur sa vie que pourrait lui conférer un statut de mère habitant avec ses enfants. Les arrangements au sein du couple lui donnent la possibilité d'avoir une activité salariée. Ce «studio » apparaît pour Fatoumata comme « un support d'activités émancipatrices » hors du rapport salarial (Lambert, 2016, p. 58), comme l'illustrent par exemple son activité commerciale ou encore le réseau de sociabilités et de solidarité qu'elle y a tissé.

\section{Les ressources sociales nécessaires pour avoir un chez-soi en dehors de la cohabitation conjugale}

Sur l'ensemble des femmes que j'ai rencontrées dans le cadre de l'enquête, un certain nombre d'entre elles n'a jamais fréquenté les dispositifs du « 115 ». Quelles en sont les conditions de possibilité ? Quelles sont les autres formes de domination qui s'expriment dans ces trajectoires et dans leurs espaces domestiques ? Ces femmes mieux dotées socialement, semblent jouer de leurs capitaux sociaux et économiques pour s'épargner l'expérience déclassante des centres d'hébergement. La possession d'un chez-soi où elles vivent seules, permet également à ces femmes de choisir de ne pas vivre en cohabitation conjugale et de se soustraire aux regards masculins.

\subsection{Les capitaux garants du chez-soi}

Je rencontre Lalia à l'association « Droits des sans-papiers ». Notre relation est marquée par une forte proximité sociale : Lalia, âgée de 30 ans est fortement politisée depuis l'université en Algérie, elle est passionnée de littérature et diplômée d'un Master. En France depuis 2009, je la rencontre au début de sa troisième année de «sans-papiers". Ses parents lui rendent visite chaque été. Je prends réellement conscience de la position sociale de Lalia lors de notre rencontre dans son appartement.

\subsubsection{Vivre dans un quartier bourgeois}

Lalia vient me chercher à la station de métro d'un quartier bourgeois du centre de Paris. Des enseignes de luxe dans le prêt-à-porter, d'autres enseignes davantage en direction des classes moyennes sont établies dans le quartier. À cette heure (début d'après-midi), les gens se croisent : touristes, habitant.e.s du quartier et travailleurs.ses. La hiérarchie dans la division du travail s'exprime dans les tenues et le maintien des corps : entre les employé.e.s des boutiques et les cadres, 
par exemple. Lalia me parle de ce quartier qu'elle apprécie, mais y regrette l'absence de commerces alimentaires. Elle fait ses courses à « Barbès ». Pourtant, quelques commerces d'épicerie fine sont présents mais leurs prix lui rendent leur fréquentation impossible. Son appartement se situe dans une petite rue peu fréquentée. Une grande porte cochère verte marque l'entrée de son immeuble haussmannien. Des plantes bien entretenues habillent une grande cour pavée. Une première porte dessert un vaste hall avec un ascenseur et la loge de la concierge. Au fond de la cour une autre porte, un hall sans ascenseur et un escalier du type de ceux réservés autrefois aux chambres de bonnes, quoique plus vaste, nous amène à son appartement situé au troisième étage. Les murs du couloir sont clairs et bien entretenus.

\subsection{2 "Mon studio » : des éléments de décor et un goût de la petite bourgeoisie culturelle}

La pièce est vaste (plus de $25 \mathrm{~m}^{2}$ ), très haute de plafond. Une grande fenêtre éclaire la pièce et ses murs blancs. Le plancher est recouvert de moquette. Une petite salle de bains renferme la douche et les toilettes. Seule une horloge est accrochée aux murs. Lalia me décrit d'elle-même le lieu où elle vit. Il y a d'abord un canapé, sous la fenêtre qu'elle convertit en lit la nuit. Acheté à Ikea, c'est « un ami de la fac d'Algérie » qui l'a aidée à le transporter. À côté du lit, une table de nuit sur laquelle repose une poupée en porcelaine ancienne. Une table basse, sur laquelle sont posés ce jour de nombreux gâteaux et du jus de fruit. Sa machine à laver a été achetée sur le site leboncoin.fr. Sa tante lui a donné un petit four. À la description de cet objet Lalia tient à me préciser qu'elle n'aime pas tellement cuisiner. Son studio est aussi composé de deux meubles récupérés dans la rue. Sur son bureau en métal, une télévision, un ordinateur portable et une webcam sont installés. Le matériel informatique a été donné par le père de la petite fille qu'elle garde à Pantin. Elle travaille avec cette famille pour en moyenne 650 euros par mois, sans compter les heures de garde en soirée payées en complément. Dans ce logement, elle possède un abonnement internet au nom de son cousin, qu'elle rembourse tous les mois. Les photos qu'elle me montre décrivent ses vacances à Étretat et à Marseille. On y voit également les après-midis de week-ends à discuter avec des amis sur la pelouse du parc de Montmartre, celui de la Villette, au Jardin du Luxembourg. Puis, il y a les soirées organisées à l'occasion de mariage, les concerts au centre culturel algérien, les débats politiques. Ces photos dénotent un réseau amical et familial fort et structurant. Lors de ma visite, elle sort du dessous de son armoire son tapis de prière. Lalia se définit comme "un peu pratiquante ». C'est par son oncle " avocat d'affaire » en Algérie qu'elle obtient son studio. Un ami de celui-ci, propriétaire du studio le loue à Lalia pour 750 euros par mois. Le bail est au nom de Lalia mais c'est son oncle qui paye le loyer.

Les mots de Lalia sont toujours passionnés. Au cours de ses récits sur la situation politique en Algérie ou sur la Kabylie, elle va chercher quelques livres qui l'ont toujours accompagnée dans son parcours d'adolescente à l'âge adulte. Elle lit Albert Camus, Kateb Yacine et d'autres. Elle aime la poésie. Cette lectrice 
qui écrit depuis l'âge de ses 14 ans fréquente une bibliothèque municipale et y emprunte des livres.

Que nous apprend sur sa position sociale ce capital culturel non certifié qui s'exprime par le biais de son studio, des objets qui y sont disposés et de ses pratiques culturelles? Lalia a connu une ascension sociale par rapport à ses parents : ses titres scolaires - universitaires - et son emploi de journaliste dans un quotidien national à Alger ${ }^{18}$ l'illustrent. Elle a une érudition, un rapport à la lecture et des sorties culturelles caractéristiques de la petite bourgeoisie culturelle, tout en s'en distinguant sur certains points. Certaines de ces formes de culture sont effectivement aux marges de la culture légitime en France et en Algérie (auteurs kabyles, musiciens et chanteurs kabyles). Ce rapport à la lecture a été transmis par sa famille et renforcé par son parcours universitaire et sa socialisation politique. Son appartenance kabyle, fortement revendiquée, est l'héritage d'une mémoire familiale et collective qui s'est davantage affirmée dans ses engagements associatifs et militants politiques à Alger. Ces engagements ont fortement structuré son quotidien d'étudiante. Les sociabilités militantes et les formes d'injonction à la culture politique (lecture de la presse et littérature militante, mise en exergue de la biographie de personnalités, etc.) ont favorisé "l'incorporation de manière d'être, de parler par imprégnation » (Pudal, 1989, cité par Darmon, 2010 [2006], p. 86). Sa socialisation politique et son capital scolaire participeront à lui offrir une position importante à Paris dans l'association «Droits des Sans-Papiers » où je l'ai rencontrée. Vivre seule me dit Lalia, lui donne la possibilité d'échapper aux tâches domestiques qu'auraient nécessité une cohabitation conjugale et l'organisation du repas familial. Pour Lalia, la figure repoussoir est celle de l'épouse, femme au foyer. L'activité salariée des femmes hors du foyer constitue pour elle un élément de l'émancipation des femmes et construit par là même les tâches domestiques comme des tâches ingrates. Cette norme de l'activité féminine participe à hiérarchiser les femmes entre elles dans le discours de Lalia, agissant comme un signe distinctif ${ }^{19}$, reflet d'une lutte de classement. En se positionnant ouvertement dans son discours à distance des tâches domestiques, elle se place du côté des classes moyennes et supérieures.

Par le biais de ce portrait de Lalia, j'ai souhaité montrer le lien de causalité entre son capital social, culturel et économique et son choix de résidence. Elle peut, dans son quartier bourgeois se tenir géographiquement à distance des classes populaires. Elle peut échapper aux violences sociales des déclassements subis dans les centres d'hébergement. Elle fait partie des catégories sociales les plus hautes des interlocutrices sans-papiers que j'ai rencontrées qui n'ont jamais eu à subir la perte d'un chez-soi. Son mode de vie est assez proche d'un mode de vie estudiantin à Paris, si l'on ne tient pas compte de la fragilité de sa situation

18 Lalia est née dans une ville moyenne de Kabylie au sein d'une famille de trois enfants (une fille et deux garçons) dont elle est la cadette. Ses parents, aujourd'hui à la retraite étaient tous deux enseignants.

19 Ces réflexions font écho aux analyses d'Anne Lambert (2016, p. 68) à propos des ménages engagés dans une trajectoire sociale ascendante habitant dans un lotissement pavillonnaire. 
administrative de sans-papiers. Jeune, elle peut encore se penser comme une adulte en devenir, dans un mode transitoire. Elle pense que ses diplômes, son origine sociale, lui ouvrent ici à Paris, l'espoir d'une vraie mobilité sociale pour intégrer les couches culturelles de la petite bourgeoisie. L'âge est aussi une variable sociale décisive pour comprendre les trajectoires de ces femmes.

\subsection{L'espace des domestiques réinventé}

Nadra que j'ai rencontrée à l'association Droits des Sans-Papiers me reçoit dans sa chambre de bonne située au sixième étage d'un immeuble bourgeois du $\mathrm{XVI}^{\mathrm{e}}$ arrondissement. Auparavant elle était logée chez sa cousine puis chez son employeuse. Payée par cette dernière 1000 euros par mois, elle a pu économiser pour commencer à louer cette chambre de bonne. Son amie, concierge dans le $\mathrm{XVI}^{\mathrm{e}}$ arrondissement et rencontrée par le biais des cours de français, lui trouve par son réseau professionnel une chambre de bonne. Sa chambre fait $10 \mathrm{~m}^{2}$. Il n'y a pas d'ascenseur qui mène aux chambres de bonnes. Elle paye 420 euros de loyer par mois sans la taxe d'habitation, l'électricité et l'assurance. Les heures de ménage qu'elle effectue, principalement dans le $\mathrm{XVI}^{\mathrm{e}}$ arrondissement, lui permettent d'assurer ce loyer, ses dépenses quotidiennes, quelques consommations pour «se faire plaisir » et d'envoyer de l'argent à «sa mère biologique $»^{20}$.

\subsubsection{Filmer pour dénoncer le désintérêt des bourgeois}

Nadra a fait plusieurs petits films sur son logement. Ils ont été réalisés en février 2011 dans le cadre de l'atelier vidéo participatif mis en place à Droits des Sans-Papiers avec des amis. Nous lui avons confié une petite caméra qu'elle a gardée avec elle plusieurs semaines pour filmer, si elle le souhaitait, l'endroit où elle vivait. Aussi, ces images prennent corps dans un contexte particulier : elle filme pour moi, pour nous. Elle veut révéler les conditions sociales dans lesquelles elle se sent maltraitée. L'ordre retenu pour ces images et présenté ici suit celui des films de Nadra.

Ces images rendent compte de deux registres distinctifs dans le même immeuble. Il y a l'ordre bourgeois d'espace commun, l'espace de la copropriété, tout est rangé, à sa place, en ordre. Une mise en scène de la distinction bourgeoise peut être notée dans l'entrée et dans la cour : l'architecture classique est mise en valeur, le lustre, la grande porte cochère en bois massif, les plantes sont toutes dans des pots disposés en ordre, le quadrillage vert accueille des plantes grimpantes, le calme, le repos de l'entre-soi bourgeois (photographie 2). Ces prises de vues de Nadra sont faites très rapidement. Nadra ne parle pas, une attitude qui souligne que cet espace est réservé : elle peut juste le traverser le plus rapidement possible car elle n'y est pas à sa place, elle n'est pas chez elle tant qu'elle n'est pas dans

20 Issue des classes populaires, Nadra née en 1978 a grandi à Casablanca. Suite à la mort de son père elle est confiée à son oncle paternel et devient " petite bonne » au sein l'unité domestique. Puis, " reprise » et hébergée par sa tante (la sœur de son père qu'elle appelle " ma mère adoptive »), elle apprend le métier de brodeuse. Elle arrive en France en 2002. 


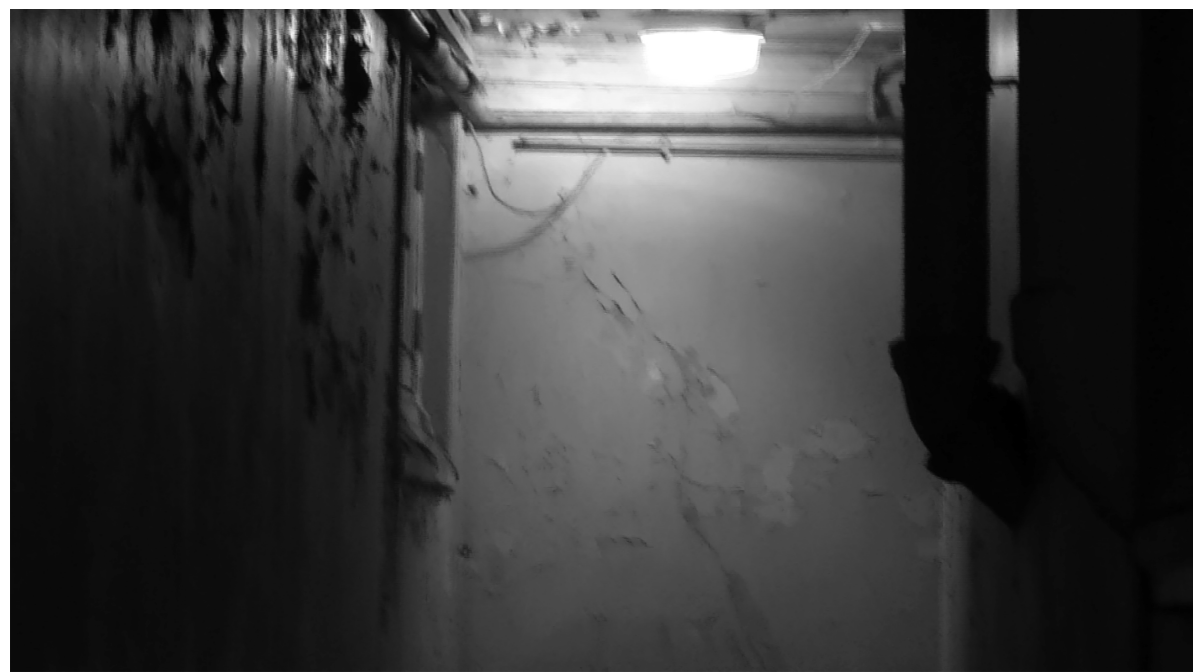

Source : cliché de Nadra, 2011

Fig. 2 Nadra fait un gros plan sur le plafond pour en souligner l'état de délabrement. Nadra's close-up of the ceiling taken to highlight the dilapidated conditions.

l'escalier de service. Elle semble hors du bon ordonnancement des lieux. Puis on découvre l'escalier de service, une autre entrée presque cachée, l'entrée des classes populaires principalement racisées, alors que nous sommes toujours dans le même immeuble. Les prises de vues de Nadra sont alors beaucoup plus longues. Nadra filme les murs, le plafond, la peinture qui se décolle, des câbles qui pendent. Elle fait de gros plans sur la peinture écaillée, la lumière blafarde. Cette partie de l’immeuble est négligée par le syndic et la copropriété, une négligence qui fait une violence aux classes populaires racisées qui l'habitent, comme me l'explique Nadra lors de l'entretien. Lorsque je lui demande pourquoi elle avait voulu filmer l'entrée de l'immeuble, nous montrer les « deux entrées différentes », elle me dit :

«Parce que [...] cette entrée [l'entrée principale] elle est tout le temps propre, $[\ldots]$ tu l'as vu, nickel, et tu l'as vu l'entrée de l'escalier [celle des chambres de bonnes] ? Y'a une grosse, grosse différence non ? [...] [I]ls font pas de travaux dans l'escalier depuis longtemps, je ne sais pas combien... Des années, même pour les couloirs! »(Entretien, le 4 mars 2012 chez Nadra)

Puis elle me demande : "Et pourquoi à ton avis ils ne font pas ces travaux ?» Pour Nadra, c'est l'appartenance "étrangère » des locataires des chambres de bonne. Selon elle, une « Française » serait plus offensive que les « étrangers » car les "étrangers ils veulent pas de problèmes". Comme par une inertie du bâti, ces femmes d'aujourd'hui occupent toujours les espaces des domestiques d'hier, les « chambres de bonnes », mais ne sont plus bonnes des bourgeois.es de l'immeuble 
(le rapport de domesticité entre les occupant.e.s des chambres et les résident.e.s bourgeois.es a disparu). La division du travail n'a pas changé, les bourgeois.es ont toujours des personnels à leur service, mais leur répartition spatiale dans la ville a changé. Les rapports de domination sociale dans l'espace résidentiel partagé ne sont plus enchâssés dans une relation de service, d'employeur.se à employé.e, une relation de domination personnalisée. Sans fonction sociale dans ces lieux bourgeois, ces occupant.e.s des chambres de bonnes sont à forte distance sociale de leurs co-résident.e.s et la domination sociale née de la proximité spatiale est désormais impersonnelle (Chamboredon, Lemaire, 1970). Face à cette violence d'être déclassée par le groupe avec lequel elle co-réside, l'intérieur de Nadra, décoré, agencé avec soin apparaît comme une restauration et une protection de soi. Elle aussi a divisé l'espace de la chambre en plusieurs coins ou sous espaces thématisés (Bony, 2015) : " le coin cuisine », le coin bureau, la douche, " ma p'tite table », etc.

Nadra apprécie de vivre seule et ne souhaite pas vivre en cohabitation conjugale, ce que lui permettent la location de sa chambre et son emploi :

«Des Marocains, si j’en ai rencontré mais je ne sais pas, je me suis habituée à être toute seule alors ça c'est plus difficile [...]. C'est difficile surtout pour quelqu'un qui est habitué à habiter seule depuis cinq ans, même plus. » (Entretien, le 4 mars 2012 chez Nadra)

Le studio de Nadra est ici un espace de résistance, il lui permet de se soustraire au regard extérieur et au contrôle du ou des regards masculins que pourrait impliquer une cohabitation conjugale.

\subsubsection{Domination personnalisée avec les fractions établies des classes populaires}

À plusieurs reprises, pendant et hors de l'entretien, elle évoque les tensions rencontrées avec la concierge de l'immeuble. Cette concierge, me dit Nadra, «veut que je nettoie mais je nettoie pas. Je nettoie ma chambre et c'est tout parce que je paye mon loyer, c'est plus cher que les autres ». Selon Nadra les autres locataires de l'étage ont un loyer de 300 euros. La concierge sait que Nadra n'a «pas de papiers » et « essaye d'en profiter» selon elle. La concierge qui représente ici les classes populaires établies, en raison de son rapport au salariat et au statut administratif, semble entretenir une relation de domination avec les locataires des chambres de bonnes. Sans recours aux droits, ces interlocutrices peuvent être exploitées par les classes populaires établies. Nadra aimerait trouver un autre logement en raison des escaliers qui mènent à sa chambre de bonne, «c'est fatiguant» dit-elle. Seulement, l'emplacement de sa chambre est idéal, elle souhaite garder une position résidentielle centrale pour réduire ou conserver une faible distance au travail, une stratégie qui souligne son ascension sociale (Le Bars, 2017). 


\section{Conclusion}

Des travaux récents ont montré combien l'espace domestique pouvait être un espace de résistance pour les femmes de différents groupes sociaux et qu'il était porteur de pratiques contestataires (Lambert, 2016). Ces réflexions participent à travailler les représentations classistes qui pouvaient être portées par les féministes et plus largement les chercheurs.ses sur les femmes des classes populaires les voyant comme des "ménagères conservatrices » (Marx Ferree, 1980) ${ }^{21}$ comme l'ont souligné les féministes anglophones ${ }^{22}$. C'est dans ce sillage que cet article a cherché à s'inscrire.

Se pencher sur le logement de femmes sans-papiers donne à voir une expérience genrée et racisée de la ville où le système de contraintes sur ces femmes est fort. J'ai d'abord voulu souligner la façon dont l'instance institutionnelle, celle des dispositifs du « 115 » intervient de manière significative sur les pratiques, sur les corps de femmes sans-papiers et rend impossible la construction d'un chez-soi. Puis, à l'échelle du chez-soi, aussi petit et précaire soit-il, comme pour Fatoumata avec "son château » et Nadra avec "sa chambre de bonne ", son aménagement, sa décoration, les objets et meubles que l'on y entrepose mais aussi la façon dont on en parle apparaissent comme des techniques d'appropriation et de protection. Et parfois, à l'échelle de l'immeuble, du quartier, des formes d'ancrage et de ressources s'élaborent. Cet article permet également d'identifier les variables sociales discriminantes des trajectoires résidentielles et de la construction d'un chez-soi de femmes sans-papiers (capital social, culturel, économique, âge, statut matrimonial, réseaux familiaux et amicaux, socialisation). L'appropriation de l'espace domestique et les modes d'investissement dans celui-ci nous donnent à voir combien genre, « race » et classes se forgent au quotidien pour définir les positions sociales de ces femmes.

Les ressources sociales initiales, même maigres, différencient nettement les trajectoires de celles qui sont pourtant censées n'avoir plus rien.

Cité Descartes - Bâtiment Bienvenüe - Plot A

14-20 Boulevard Newtown

Champs-sur-Marne

77454 Marne-la-Vallée cedex 2

lebarsjoanne@gmail.com

21 Traduction de l'anglais par Anne Lambert (2016).

22 Je renvoie également à ce sujet aux travaux de Beverley Skeggs (2015), ceux de Judith Stacey (1998 [1990]) et à ceux du Women's Studies Group (1978). 


\section{Bibliographie}

Béguin, H. (2015), « Héberger des migrants ou gérer des logements ? L'Aftam et ses "foyers d'Africains noirs" (1962-2012) ", thèse de doctorat en urbanisme, aménagement et politiques urbaines, université Paris-Est, 530 p.

Bensa, A. (2006), La fin de l'exotisme. Essais d'anthropologie critique, Toulouse, Anacharsis, 364 p.

Bernardot, M. (2008), « Camps d'étrangers, foyers de travailleurs, centres d'expulsion : les lieux communs de l'immigré décolonisé », Cultures \& Conflits [En ligne], vol. 69, consulté le 30 septembre 2016.

Bony, L. (2015), "La domestication de l'espace cellulaire en prison », Espaces et sociétés, vol. 3, $\mathrm{n}^{\circ} 162$, p. 13-30.

Bouillon, F. (2005), " Le squat une alternative à la rue ? » in BRODY J. (dir.), La rue, Toulouse, Presses universitaires du Mirail, p. 179-194.

Bouillon, F. (2011), "Awa, une Malienne à Paris », in Agier (dir.), Paris refuge. Habiter les interstices, Broissieux, Croquant, p. 165-189.

Bourdieu, P. (1979), La distinction. Critique sociale du jugement, Paris, Éditions de Minuit, 672 p.

Bourdieu, P. (1978), «Classement, déclassement, reclassement », Actes de la recherche en sciences sociales, vol. 24, p. 2-22.

Cefaï, D., Gardella, É. (2011), L'urgence sociale en action : ethnographie du Samu social de Paris, Paris, La Découverte, $750 \mathrm{p}$.

Chamboredon, J.-C., Lemaire M. (1970), «Proximité spatiale et distance sociale. Les grands ensembles et leur peuplement ", Revue française de sociologie, vol. 11, n 1, p. 3-33.

Chamboredon, J.-C. et al. (2009 [1985]), «L'appartenance territoriale comme principe de classement et d'identification », in Weber F., Le métier d'ethnographe, Paris, PUF, p. 152-171.

Collignon, B., Staszak, J.-F. (2004), Espaces domestiques. Construire, habiter, représenter, Paris Bréal, $447 \mathrm{p}$.

Darmon, M. (2010), La socialisation, Paris, Armand Colin, 128 p.

Delage, M., Weber, S., 2014, "L'espace résidentiel des étrangers dans la métropole parisienne ", Hommes \& migrations, $\mathrm{n}^{\circ} 1308$, p. 13-26.

Drouilleau, F. (2009), «Exode et domesticité à Bogotá », Travail, genre et sociétés, vol. 2, n 22, p. 75-96.

Fainzang, S., Journet, O. (1988), La femme de mon mari, Paris, L'Harmattan, 174 p.

Feldman, N. (2013), «Division sexuelle du travail et mobilités géographiques féminines », Géocarrefour, vol. 88, n 2, p. 97-106.

Fischer, N. 2010. "Les corps-frontières. Atteinte physique et expertise médicale dans un centre de rétention administrative ", in Fassin D., Fassin É., (dir.), Les nouvelles frontières de la société française, Paris, La Découverte, p. 477-498.

Fol, S. (2010), "Mobilités et ancrages dans les quartiers pauvres: les ressources de proximité », Regards Sociologiques, $\mathrm{n}^{\circ} 40$, p. 27-43.

Fraisse, G. (1979), Femmes toutes mains. Essai sur le service domestique, Paris, Le Seuil, 246 p.

François, C. (2014), «Au mépris des locataires. Infériorisation sociale et assignation résidentielle des relogés de la rénovation urbaine », Genèses, 2014, vol. 3, nº 96, p. 86-109.

Girard, V. et al. (2013), "Propriétés et classes populaires : des politiques aux trajectoires », Politix, vol. 1, n 101, p. 7-20.

Gilbert, P. (2016), "Classes, genre et style de vie dans l'espace domestique », Actes de la recherche en sciences sociales, vol. $15, \mathrm{n}^{\circ} 125$, p. 4-15. 
Girola, C. (2011), Vivre sans abris. De la mémoire des lieux à l'affirmation de soi, Paris, rue d'ULM, $68 \mathrm{p}$.

Goffman, E., (1973), La mise en scène de la vie quotidienne. La présentation de soi, Éditions de Minuit, 256 p.

Hmed, C. (2006), "Tenir ses hommes". La gestion des étrangers "isolés" dans les foyers Sonacotra après la guerre d'Algérie », Politix, vol. 4, n 76, p. 11-30.

Laé, J.-F., Proth B. (2002), "Les territoires de l'intimité, protection et sanction », Ethnologie française, Vol. 32, $\mathrm{n}^{\circ} 1$, p. 5-10.

Lambert, A., Dietrich-Ragon, P., et al., 2014, Argumentaire du cycle de Journées d'étude " Femmes et habitat : une question de genre », Ined, 2014/2015.

Lambert, A. (2016), «Échapper à l'enfermement domestique. Travail des femmes et luttes de classement en lotissement pavillonnaire ", Actes de la recherche en sciences sociales, vol. 5, n 215, p. 56-75.

Le Bars, J. (2017), "Conquérir la galère », Géographie féministe postcoloniale de femmes sanspapiers venues $d^{\prime}$ Afrique subsaharienne et du Maghreb en région parisienne, thèse de doctorat en géographie, Université Paris-Est, 667 p.

Le Mener, E., Oppenchaïm, N. (2012), "The Temporary Accommodation of Homeless Families in île-de-France : Between Social Emergency and Immigration Management », European Journal of Homelessness, vol. 6, $\mathrm{n}^{\circ} 1$, p. 83-103.

Le Mener, E. (2013), "Quel toit pour les familles à la rue ? L'hébergement d'urgence en hôtel social », Métropolitiques [en ligne], consulté le 20 mai 2016, http://www.metropolitiques.eu/Quel-toitpour-les-familles-a-la.html.

Lesselier, C. (2004), "Femmes migrantes en France. Le genre et la loi », Les cahiers du CEDREF, vol. 12 , p. 45-59.

Lion, G. (2014), "En quête de chez-soi. Le bois de Vincennes, un espace habitable? », Annales de Géographie, vol. 3, n 697, p. 956-981.

Mahmood, S. (2009), Politique de la piété : le féminisme à l'épreuve du renouveau islamique, Paris, La Découverte, 311 p.

Makaremi, C. (2009), Zone d'attente pour personnes en instance. Une ethnographie de la détention frontalière en France, thèse de doctorat en anthropologie, Université de Montréal, 426 p.

Marx Ferree, M. (1980), "Working Class Feminism: a Consideration of the Consequences of Employment », The Sociological Quaterly, vol. 21, p. 173-184.

Martin-Fugier, A. (1985), La place des bonnes, Paris, Livre de Poche, 405 p.

Mazouz, S. (2007), "Les mots pour le dire », Recherches, p. 81-98.

Mbodj-Pouye, A. (2016), " "On n'ignore pas la solidarité". Transformation des foyers de travailleurs migrants et recompositions des liens de cohabitation », Genèses, vol. 3, n 104, p. 51-72.

McDowell, L. (2004 [1999]), Gender, Identity \& Place. Understanding feminist geographies, Cambridge, Polity Press, 284 p.

Moss, P., Dyck, I. (2003), Women, body, illness: space and identity in the everyday lives of women with chronic illness, Oxford, Rowman \& Littlefield, 225 p.

Moujoud, N. (2007), Migrantes, seules et sans droits, au Maroc et en France. Dominations imbriquées et résistances individuelles, thèse de doctorat en anthropologie sociale et ethnologie, École des Hautes Études en Sciences Sociales de Paris, 521 p.

Moujoud, N. (2011), Montagne, migration post-coloniale et domesticité de jeunes filles au Maroc, communication présentée « journée scientifique organisée par Pôles Suds (Ined) », Ouagadougou, [enligne], http://pole_suds.site.ined.fr/fichier/s_rubrique/20591/moujoud.fr.pdf

Nicollet, A. (1992), Femmes d'Afrique noire en France. La vie partagée, Paris, L'Harmattan, 317 p. 
Oso Casas, L. (2005), "La réussite paradoxale des bonnes espagnoles de Paris », Revue européenne des migrations internationales, vol. 21, $\mathrm{n}^{\circ} 1$, p. 107-129.

Pan Khé Shon, J.-L., Robello S. (2010), «Inégalités des transitions de logement, discriminations et ségrégations perçues », in Trajectoires et Origines. Enquête sur la diversité des populations en France, Documents de travail, $\mathrm{n}^{\circ} 168$, Ined, p. 95-100.

Paugam, S. (2004 [1991]), La disqualification sociale. Essai sur la nouvelle pauvreté, Paris, PUF, 256 p. Perrot, M. (2009), Histoire de chambres, Paris, Seuil, 444 p.

Poiret, C. (1996), "L'inclusion des familles africaines en Île-de-France : de la catégorie "ethnique" aux groupes "africains" ", Espace, populations, sociétés, vol. 14, n 2, p. 335-346.

Pratt, G. (2004), Working Feminism, Philadelphia, Temple University Press, 224 p.

Razy, É. (2006). "Les migrants ont-ils des manières particulières d'habiter ? L'exemple soninké ", Hommes \& migrations, $\mathrm{n}^{\circ} 1264$, p. 77-87.

Samuel, M. (1978), Le prolétariat noir en France, Paris, Maspero, 262 p.

Sayad, A. (1980), " Le foyer des sans-famille », Actes de la recherche en sciences sociales, vol. 32-33, p. 89-103.

Schmoll, C., 2005, "Pratiques spatiales transnationales et stratégies de mobilité des commerçantes tunisiennes », Revue Européenne des Migrations Internationales, vol. 21, nº 1, p. 131-154.

Schwartz, O. (2012), Le monde privé des ouvriers, Paris, PUF, 552 p.

Silvey, R. (2004), "Power, difference and mobility: feminist advances in migration studies », Progress in Human Geography, vol. 4, n² 28, p. 490-506.

Simon, P. (1995), «Le logement des immigrés », Population et sociétés, n 303, p. 1-4.

Skeggs, B. (2015), Des femmes respectables. Classe et genre en milieu populaire, Paris, Agone, 432 p.

Stacey, J. (1998 [1990]), Brave New Families. Stories of Domestic Upheaval in Late Twentieth Century America, Berkeley, University of California Press, 328 p.

Têtu-Delage, M.-T. (2009), Clandestins au pays des papiers : expériences et parcours de sans-papiers algériens, Paris, La Découverte, 244 p.

Thalineau, A. (2002), "L'hébergement social : espaces violés, secrets gardés », Ethnologie française, vol. 1, n³2, p. 41-48.

Timera, M. (1997), "Sans-papiers africains face aux "communautés" d'origine », in FASSIN D. et al. (dir.), Les lois de l'inhospitalité. Les politiques de l'immigration à l'épreuve des sans-papiers, Paris, La Découverte, p. 95-106.

Tuan, Y.-F. (2014 [1977]), Space and Place. The perspective of experience, University of Minesota Press, $235 \mathrm{p}$.

Verdugo, G. (2011), « Logement social et ségrégation résidentielle des immigrés en France, 1968$1999 »$, Population, vol. 66, n 1, p. 171-196.

Weber, F. (2013), Penser la parenté aujourd'hui. La force du quotidien, Paris, Rue d’Ulm, 264 p.

Women's Studies Group, 1978, Women Take Issue. Aspects of Women's Subordination, London, Hutchinson, $216 \mathrm{p}$.

Yaouancq, F. et al. (2013), L'hébergement des sans-domicile en 2012. Des modes d'hébergement différents selon les situations familiales, Paris, Insee.

Zeneidi-Henry D. (2002), Les SDF et la ville. Géographie du savoir-survivre, Paris, Bréal, 288 p. 